\title{
La résidence en pharmacie au Canada : Possibilités et nouveaux défis
}

\author{
par Peter J Zed
}

$\mathrm{D}$ epuis plus de 40 ans, les programmes de résidence en pharmacie d'une année ont été la pierre angulaire de la formation visant à compléter le baccalauréat des pharmaciens intéressés à poursuivre une carrière en pharmacie hospitalière. Le Conseil canadien de la résidence en pharmacie d'hôpital $(\mathrm{CCRPH})$ définit la résidence en pratique pharmaceutique comme « un programme organisé, dirigé et agréé qui renforce les compétences acquises dans un programme professionnel de premier cycle agréé en pharmacie. La résidence en pratique pharmaceutique est axée sur les soins directs aux patients, les activités de la pharmacie, la gestion de projet et l'aspect expérience personnelle de la profession de pharmacien $»^{1}$. Les bacheliers des programmes de pharmacie actuels ne sont pas préparés pour fonctionner de façon autonome dans les environnements de la pharmacie hospitalière d'aujourd'hui, où ils doivent prodiguer des soins directs aux patients et répondre aux attentes d'un champ de pratique en constante évolution. Bien que la portée et les activités des programmes actuels de résidence en pratique pharmaceutique soient différents, ces programmes comblent néanmoins l'écart entre les connaissances et l'expérience acquises dans le cadre des études de premier cycle et celles de la pratique en pharmacie hospitalière, et préparent mieux les pharmaciens à la pratique en milieu hospitalier.

La pratique de la pharmacie et les attentes envers les pharmaciens des milieux hospitaliers ont évolué de concert. Plus que jamais, les pharmaciens d'hôpitaux ont l'occasion de mettre à profit l'ensemble de la sphère de leurs compétences, ce qui cependant se traduit par des attentes plus élevées envers ceux qui ont terminé un programme de résidence en pratique pharmaceutique. D'ailleurs, certains ont remis en question l'approche et la durée de la formation qui suit le baccalauréat et qui vise à préparer les pharmaciens à l'exercice de leur profession en milieu hospitalier, comme en témoignent plusieurs nouveaux programmes de pratique pharmaceutique d'une durée de 24 mois plutôt que de 12 mois aux États-Unis ${ }^{2}$. L'American
Society of Health-System Pharmacists et l'American College of Clinical Pharmacy ont tous deux défini le programme de résidence en pharmacothérapie de 24 mois comme étant " conçu pour former des praticiens spécialisés dans la prestation de soins pharmaceutiques à diverses populations de patients hospitalisés ou ambulatoires présentant des problèmes de santé aussi variés que complexes ${ }^{2}$ ». La première année de résidence permet d'accroître les compétences générales du résident en matière de gestion des systèmes de distribution des médicaments et de favoriser l'atteinte de résultats pharmacothérapeutiques optimums dans tout un éventail d'affections. La deuxième année de résidence est conçue pour approfondir les connaissances, les compétences et les habiletés du résident, renforcer ses attitudes et accroitre son degré d'expertise en gestion de la pharmacothérapie et son leadership clinique. On croit que les pharmaciens qui ont terminé la première année d'un programme de résidence de deux ans aux États-Unis, comparable à la formation des diplômés de tous les programmes au Canada, possèdent les compétences minimales requises pour fournir des services généraux, mais dans bien des cas, ils ne sont pas suffisamment autonomes pour s'acquitter de leurs responsabilités en ce qui a trait à la prise de décisions plus complexes inhérentes au choix et à la gestion de la pharmacothérapie ${ }^{3}$. La deuxième année de résidence permet aux résidents d'approfondir leurs connaissances en travaillant dans des domaines de pratique spécialisés et distincts.

Le projet SCPH 2015, dont la date butoir arrivera dans six ans, a mis de l'avant des buts et des objectifs très importants en matière de pratique de la pharmacie dans les établissements de santéf. Les pharmaciens sont appelés à élever le degré auquel les patients parviennent à une meilleure utilisation des médicaments, à mettre activement en application les méthodes fondées sur des données probantes afin d'améliorer la pharmacothérapie, à rendre l'utilisation des médicaments plus sécuritaire, à employer efficacement la technologie, et à participer à des interventions de santé publique au nom de leurs collectivités. L'un des objectifs du projet veut que d'ici 2015 
tous les nouveaux pharmaciens qui commenceront à exercer dans des établissements de santé aient terminé un programme de résidence en pratique pharmaceutique agréé par le CCRPH. Cet objectif reconnait clairement que les attentes et le champ de pratique en constante évolution dans les établissements de santé nécessitent maintenant une formation supplémentaire, au-delà du diplôme de premier cycle en pharmacie. Bien qu'on puisse s'attendre à certaines variations dans la capacité d'atteindre cet objectif, je crois que la plupart des établissements de santé canadiens n'atteindraient pas cet objectif présentement.

Dans ce numéro du JCPH, les auteurs de " Le pour et le contre " proposent deux points de vue sur la durée optimale des programmes de résidence en pratique pharmaceutique au Canada $^{5,6}$. Ils décrivent les avantages et les inconvénients d'un programme de 24 mois, étant donné l'environnement et le champ de pratique actuels de la pharmacie, ainsi que les tendances de l'enseignement de la pharmacie au Canada. En revanche, il ne faudrait pas perdre de vue la capacité actuelle et future de former des résidents en pratique pharmaceutique au Canada dans nos discussions sur cet important sujet. Lévolution de l'enseignement de la pharmacie au Canada et l'élargissement additionnel du champ de pratique des pharmaciens d'hôpitaux continueront de modeler la durée et la portée de nos programmes de résidence en pharmacie hospitalière. Malgré cela, le débat sur la durée des programmes de résidence en pratique pharmaceutique au Canada ne doivent pas occulter le défi encore plus global et certes plus important, de former suffisamment de pharmaciens dans le cadre de programmes de résidence en pratique pharmaceutique agréés pour atteindre l'objectif du projet SCPH 2015.

Les enjeux intéressant divers aspects des soins de santé surgissent rapidement et tous les fournisseurs de soins de santé doivent relever plus de défis que jamais auparavant. Les pharmaciens doivent s'ouvrir à ces changements et relever les défis de l'environnement actuel des soins de santé, en commençant par leur préparation à exercer leur profession. Peu importe la durée des programmes de résidence en pratique pharmaceutique, les établissements de santé, les régies régionales de la santé, les universités et le CCRPH doivent avant tout travailler ensemble à déterminer comment on peut former suffisamment de pharmaciens d'hôpitaux au Canada et permettre à ces professionnels hautement qualifiés de s'acquitter de leurs fonctions au plein potentiel de leur champ de pratique. Nous le devons à notre profession, et encore plus à nos patients.

[Traduction par l'éditeur]

\section{Références}

1. Société canadienne des pharmaciens d'hôpitaux, Conseil canadien de la résidence en pharmacie d'hôpital. Normes d'agrément : Janvier 2010 [Internet]. Ottawa $(\mathrm{ON})$ : Société canadienne des pharmaciens d'hôpitaux; 2009 [consulté le 11 février 2009]. Publié à http://www.cshp.ca/ programs/residencyTraining/FRENCH_-_2010_CHPRB_Accreditation_ Standards.pdf (accès réservé aux membres).

2. American College of Clinical Pharmacy; Burke JM, Miller WA, Spencer AP, Crank CW, Adkins L, Bertch KE, et al. Clinical pharmacist competencies. Pharmacotherapy 2008;28(6):806-815.

3. Nappi J, Haase K, Kessels A, Fink J. What is a pharmacotherapy residency? Benefits of a 24-month program [éditorial]. Pharmacotherapy 2008;28(7): 819-820.

4. CSHP 2015 - a practice excellence initiative for pharmacists in hospitals and related healthcare settings [Internet]. Ottawa $(\mathrm{ON})$ : Société canadienne des pharmaciens d'hôpitaux; 2007 [consulté le 27 novembre 2008]. Publié à www.cshp.ca/programs/cshp2015/index_e.asp.

5. Woloschuk D. Is a 1-year residency program long enough to prepare hospital pharmacists for practice? The "pro" side. J Can Pharm Hosp 2009;62(1):43-44.

6. Yuksel $\mathrm{N}$, Hughes $\mathrm{C}$. Is a 1-year residency program long enough to prepare hospital pharmacists for practice? The "con" side. Can J Hosp Pharm 2009;62(1):44-45.

Peter J Zed, B. Sc., B. Sc. Pharm., ACPR, Pharm. D., FCSHP est coordonnateur clinique, Département de pharmacie, et spécialiste de la pharmacothérapie, Médecine d'urgence, au Queen Elizabeth II Health Sciences Centre, et professeur agrégé, Collège de pharmacie et Département de médecine d'urgence, Université Dalhousie, Halifax, Nouvelle-Écosse. Il est également rédacteur adjoint du $J C P H$.

\section{Adresse de correspondance :}

Dr Peter J Zed

Department of Pharmacy — Halifax Infirmary

Queen Elizabeth II Health Sciences Centre

1796 Summer Street

Halifax NS

B3H 3A7

courriel : peter.zed@dal.ca 